\section{Neue Angst um Honorarverlierer}

Die Honorarverteilung liegt seit Januar wieder in regionalen Händen. Doch längst nicht aus jeder KV sind Jubelschreie zu hören. Zu groß ist die Angst, dass es neue Honorarverlierer gibt.

$\mathrm{M}$ it dem VStG (Versorgungsstrukturgesetz) wurde zu Jahresbeginn die Honorarverteilung wieder auf die KVEbene verlegt. Neun KVen haben eine Einschätzung abgegeben, was die wieder gewonnene regionale Verantwortung für die Honorare an Vorteilen, aber auch an Nachteilen bringt.

Das Ergebnis: Generell stößt der Wunsch des Gesetzgebers, die Regionen wieder stärker auch in Honorarfragen zu beteiligen, bei den KVen Hamburg, Schleswig-Holstein, Berlin, Sachsen, Sachsen-Anhalt, Nordrhein, Saarland, Rheinland-Pfalz und Baden-Württemberg auf Zustimmung. Denn, so die einhellige Meinung, nun könnten wieder regionale Besonderheiten in die Honorare einfließen. Doch die Vergütungsunterschiede, die noch vor der letzten Reform beschlossen wurden, ließen sich in regionalen
Verhandlungen mit den Kassen nicht ausgleichen, bemängelt die KV Nordrhein. Noch drastischer drückt es die KV Sachsen-Anhalt aus: Da die regionale Morbidität bei der Gesamtvergütung 2012 gar nicht anerkannt werde, fürchtet die $\mathrm{KV}$, dass der Honorarverteilungsmaßstab „ein Instrument zur Verteilung des Mangels an Honorar bleibt".

Nicht alle KVen sehen so düster. „In direkten Verhandlungen mit den Krankenkassen lässt sich das Maximale herausholen“, sagt Walter Plassmann, stellvertretender Vorsitzender der KV Hamburg. Ähnliche Töne sind aus Baden-Württemberg zu hören. Bei der KV Saarland ist man hingegen noch gar nicht so sicher, ob der regionale Spielraum so groß sein wird. Weil auch mit dem VStG zunächst die KBV die Vorgaben für die Honorarverteilung festlegt.

\begin{abstract}
Abrechnungstipp
Hausbesuch im Heim - Vorsicht Falle!

- Wenn Neurologen Hausbesuche machen, sind es sicher in vielen

Fällen Besuche im Alten- oder Pflegeheim. Dabei ist eine kleine Falle in der Abrechnung zu bedenken: „Mitbesuche“ werden bei EBM- und GOÄ-Abrechnung unterschiedlich definiert. Da bei Besuchstouren oft mehrere Patienten besucht werden, kommt es in großer Zahl zu sogenannten Mitbesuchen nach Nr. 51 GOÄ beziehungsweise Nr. 01413 EBM. Wann aber ist ein Besuch ein Mitbesuch und wann ein Regelbesuch nach Nr. 50 GOÄ beziehungsweise 01410 EBM? Entscheidend für die Antwort ist die Definition „derselben sozialen Gemeinschaft" (EBM) oder "derselben häuslichen Gemeinschaft" (GOÄ)? Dieselbe häusliche Gemeinschaft setzt das Wohnen in derselben Wohnung oder in demselben Einfamilienhaus voraus. Auch in Alten- oder Pflegeheimen sind diese Voraussetzungen in der Regel erfüllt. Sollte es sich allerdings um ein Heim mit einzelnen abgeschlossenen Appartements handeln, ist es nicht dieselbe häusliche Gemeinschaft und die Besuche in verschiedenen Appartements sind alle nach Nr. 50 abrechenbar.

Deutlich weiter zu interpretieren ist der Begriff der sozialen Gemeinschaft. Hierzu zählen alle Bewohner eines Alten- oder Pflegeheims zum Beispiel mit Gemeinschaftsverpflegung oder anderen Formen des Gemeinschaftslebens. Hierbei ist es unerheblich, ob die Patienten auf derselben Etage wohnen oder nicht.

Übrigens: Bei GKV-Patienten ist neben dem Mitbesuch ein Wegegeld nicht berechnungsfähig. Bei GOÄ-Patienten wird das Wegegeld für alle Besuche nur einmal und zwar anteilig auf alle Besuche beziehungsweise Mitbesuche aufgeteilt berechnet.
\end{abstract}

Dr. med. Heiner Pasch

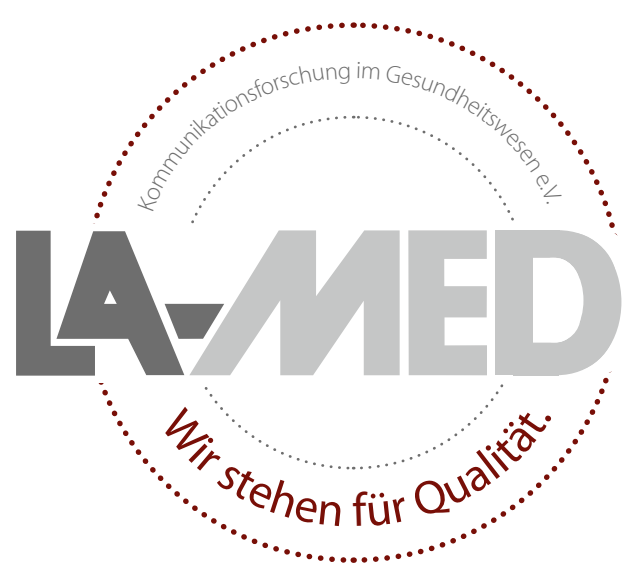

Sie lesen
Qualität

Damit das auch so bleibt, befragen wir Sie in

Kooperation mit tns emnid

in den nächsten Wochen.

Ihr Urteil ist uns wichtig.

Bitte nehmen Sie teil! 